\title{
Federalismo flexible
}

Les Metcalfe*

\section{Introducción: de}

\section{Maastricht I a Maastricht II}

Los noventa están resultando ser tiempos difíciles para la integración europea. La década se inició con la celebración de una Conferencia Intergubernamental (CIG) que concluyó en la cumbre de Maastricht, en diciembre de 1991. No obstante, en vez de consolidar los avances de los anos anteriores y alcanzar el acuerdo sobre el camino que se abría, provocó ásperas controversias, especialmente en torno al sfederalismo., al tiempo que dejó una importante cantidad de problemas sin resolver. Cuando el Tratado de la Unión Europea fue firmado a comienzos de 1992, la única alternativa posible era la de diferir a una fecha posterior algunas relevantes y conflictivas cuestiones sobre la futura forma de Europa. Esta impotencia de la CIG a la hora de resolver asuntos clave fue reconocida al incluirse en el Tratado una cláusula de revisión que preveía la celebración de otra Conferencia en 1996. La CIG 96, que rápidamente se vino en llamar Maastricht II, fue concebida para examinar en qué medida, las politicas y formas de cooperación introducidas por este tratado deberían ser revisadas con la intención de asegurar la eficacia de los mecanismos y las instituciones de la Comunidad. En otras palabras, las principales preocupaciones de Maastricht II deberían ser la eficacia y la reforma institucional.

Con el apoyo de un Grupo de Reflexión presidido por Carlos Westendorp, Maastricht II comenzó sus deliberaciones en la Cumbre de Turín, en 1996, bajo presidencia italiana y las concluyó en el Consejo Europeo de Amsterdam, en junio de 1997. El debate de la CIG evitó tocar el tema del 'federalismo", dadas las consecuencias negativas que había planteado tal término duran- te Maastricht I. Como alternativa, se concedió cierto protagonismo a la búsqueda de posibles vías que concedieran una mayor flexibilidad al funcionamiento de la Unión Europea. Federalismo versus flexibilidad es una falsa dicotomía que refleja las divisiones políticas y las diferencias ideológicas entre los Estados miembros más que las necesidades futuras de la integración europea. El propósito de este trabajo es argüir que no es posible separar flexibilidad y federalismo de esta forma. El desafío está en desarrollar nuevas formas europeas de gobernar que los combine. Las mejoras en la eficacia de la Unión dependen de la adaptación de la relación entre ellos y de desarrollar capacidades adecuadas para gestionar la integración.

El Tratado de Amsterdam, incorporando disposiciones sobre flexibilidad, fue firmado el 2 de octubre de 1997, pero esta fase del proceso de integración europea no puede darse por concluida. Existen aún riesgos sobre la ratificación antes de que el Tratado de Amsterdam pueda ocupar su lugar junto al de Roma, Maastricht y el resto de los hitos que han marcado el camino de la integración europea. Tras las fuertes reacciones contra la integración en general y el federalismo en particular durante el proceso de ratificación del Tratado de Maastricht, esta vez no deberá darse nada por sentado. Incluso si el proceso de ratificación marchara correctamente, aún quedará mucho por hacer. Aunque es demasiado prematuro realizar una evaluación final sobre la contribución de Maastricht II en el avance del proceso de la integración europea, las primeras impresiones indican un sentimiento general de decepción. Maastricht II no ha realizado un avance significativo en el tratamiento de la cuestión de eficacia. Tampoco se ha enfrentado apropiadamente a los temas de reforma institucional como se convino. Si lo evaluáramos en relación con la preparación del terreno para las tareas que han de afrontarse en el futuro, Maastricht no ha sido un éxito. Las diferencias políticas y los desacuerdos entre los Estados miembros han 
desviado los importantes retos que afrontaba la reforma. Al igual que en Maastricht I, se han dejado muchos temas inacabados. Ello no significa que haya sido un completo fracaso. Los resultados podrían haber sido incluso peores. Hasta poco tiempo antes de la cumbre de Amsterdam una crisis mayor se vislumbraba como posibilidad, debido a las agudas diferencias entre los Estados miembros y especialmente a la intransigencia del gobierno británico. No obstante, no puede dejar de reconocerse el hecho de que Maastricht II ha fracasado en su tarea de resolver algunos de los problemas estructurales más graves que necesitan ser remediados. El Tratado de Amsterdam no ha establecido una nueva base para la profundización y la ampliación de la integración europea en los próximos años. Es una omisión que tendrá un impacto muy costoso y perjudicial sobre la Unión Europea si no se rectifica pronto.

\section{Las necesidades crecientes y el "déficit de gestión"}

Se requiere urgentemente un decidido esfuerzo encaminado a la introducción de reformas institucionales y a la mejora de la eficacia de la Unión Europea (UE), debido al creciente desfase existente entre sus aspiraciones políticas y sus capacidades administrativas. La UE padece un "déficit de gestión" que, hasta tiempos recientes, apenas se había reconocido y mucho menos remediado (METCAlFE, 1995). Pese a todo, y gracias a los éxitos y a los avances de los años ochenta, no se reconocen aún la magnitud y la gravedad del problema. Desde mediados de los ochenta, el proceso de integración europea experimentó grandes avances políticos e impuso una serie de nuevas y ambiciosas metas. La "nueva dinámica. de la integración que impuso Delors durante su presidencia, tuvo como resultados el Acta Única Europea, la adhesión de España y Portugal, el programa de 1992 sobre el Mercado Único, los Tratados de Maastricht de Unión Política y Unión Económica y Monetaria y, posteriormente, la incorporación de Austria, Finlandia y Suecia. Dentro del originario pilar económico, las competencias sobre política europea devinieron mucho más amplias e importantes de lo que jamás habían sido. El Acta Única Europea y el programa del Mercado Único de 1992, incorporaron un gran elenco de aspectos económicos o relacionados con la economía, dentro del marco del proceso de la política europea, que vinieron a acelerar el proceso de integración a nivel político. En el momento en que se produce Maastricht I, la tendencia de avance se quebró, pero la UE no perdió completamente su ímpetu. El Tratado de Maastricht amplió la esfera de acción de la Unión hasta llegar a incluir una Política Exterior y de Seguridad Común en un segundo pilar y los Asuntos de Justicia y de Interior en un tercer pilar. Amsterdam ha llevado estos compromisos políticos algo más lejos y ha añadido un campo adicional de producción de políticas que deberá tratar el problema del desempleo.

¿Cuánto tiene de retórica la nueva dinámica de la integración europea y cuánto se ha traducido en resultados prácticos? Pese a haberse conseguido grandes logros, el funcionamiento del sistema aún permanece bastante por debajo de lo que se pretendía o se esperaba. Tal y como están las cosas, ninguno de los tres pilares de la Unión Europea tiene fuerza institucional suficiente para gestionar la cada vez más ambiciosa y onerosa tarea. Esta insatisfactoria situación es de general y amplio reconocimiento, pero poco se ha hecho en la práctica para remediarla. Una de las desconcertantes consecuencias es que el programa del Mercado Único está aún inacabado tras la fecha límite de 1992. Los debates inconclusos sobre las formas de organización del segundo y tercer pilar, han comprometido la eficacia en estas áreas. Se han creado Agencias europeas en varios sectores políticos como respuesta pragmática a los desajustes del sistema establecido más que como aplicación deliberada de nuevos principios de gobierno europeo. Aunque rara vez se observa desde la perspectiva de su gestión, la Unión Monetaria constituye otro ejercicio de enorme dificultad en la gestión del cambio. Además de su inherente complejidad técnica, la colosal tarea de acordar e implementar cambios tales como la creación de nuevos organismos bancarios europeos y la redefinición de las relaciones con el resto de las numerosas organizaciones financieras directa e indirectamente implicadas, ha demostrado ser extremadamente costosa. Asimismo, nos encontramos con las onerosas cargas impuestas sobre las economías, de los Estados miembros, esforzándose por alcanzar los criterios de convergencia. Se deduce de las deficiencias en estos sectores claves de la política que apenas nada ha sido realizado al objeto de constituir y desarrollar la capacidad administrativa necesaria para mantener el ímpetu de la integración o satisfacer sus cada vez más ambiciosos objetivos. La UE se enfrenta a una crisis de eficacia. Las demandas exigiendo una gestión mejor de las políticas comunitarias han aumentado y no se espera que vayan a disminuir en el futuro.

\section{Un diagnóstico del déficit de gestión}

El principal desafío que afronta la Unión Europea es el de la reducción del edéficit de gestión a través de la construcción de las capacidades necesarias para hacer frente a los actuales compromisos y a los desarrollos previsibles. Un diagnóstico sobre el déficit de gestión contribuye a desenmarañar la variedad de fac- 
tores que, reforzándose mutuamente, han permitido el surgimiento de un desfase y su crecimiento sin límite. La Comisión, como institución de la UE con la máxima responsabilidad en la gestión de las políticas comunitarias, es el punto obvio de atención en toda discusión sobre las reformas dirigidas a reducir el déficit de gestión. Pero una vez centrados en la Comisión, comienza a percibirse la dificultad en el tratamiento de los problemas. La gestión de las políticas no es la única, ni siquiera la principal, responsabilidad de la Comisión. Comparado con el mucho más brillante papel político que desempeña como iniciadora del proceso de toma de decisiones y guardiana de los Tratados, su papel gestor ha tenido siempre una menor importancia. En parte como consecuencia de ello, la Comisión misma tiene una pobre reputación como gestora. Su debilidad en el ejercicio de responsabilidades gerenciales y su preocupación central hacia el fraude y la malversación de fondos comunitarios, son bien conocidas. Administración inadecuada, pobre coordinación interna y euroburocracia han sido fáciles blancos para los críticos del proceso de integración. Un segundo aspecto del déficit de gestión es que la Comisión no tiene tradición real de reforma gerencial. A lo largo de los años esta institución ha crecido pero sin realizar ninguna reforma fundamental en su estructura o en sus mecanismos de funcionamiento. En el desarrollo de capacidades para reformas de gestión, la Comisión se ha quedado atrás con respecto a los Estados miembros, la mayor parte de los cuales se han implicado en importantes reformas de gestión en los últimos años. Durante los rápidos cambios en integración de los años ochenta, se hizo poco para proveer a la Comisión de medios para abordar sus cada vez mayores responsabilidades en materia de gestión de políticas. Delors, como presidente de la Comisión, se inclinó a adoptar la visión de que la principal tarea consistía en forzar nuevas iniciativas políticas al máximo nivel en la perspectiva de la mal fundada hipótesis de que la máquina podría hacer frente a su implementación. La consecuencia de no haber introducido las oportunas reformas de gestión en esos días, fue que la Comisión se convirtió en una organización sobrecargada y de alguna manera desmoralizada.

Otro factor a considerar es el cultural. En el campo de la integración europea, la gestión nunca ha sido una prioridad política de primer orden. Fundado en una cultura administrativa legalista que mantiene una dicotomía obsoleta entre política y administración, la gestión se identifica con la rutina de la implementación de políticas. No ha existido apenas ningún interés en el déficit de gestión, en contraste con la atención pública y la prioridad política dispensada al lanzamiento de nuevas políticas o a la reducción del déficit democrático en la UE. Los desajustes en la gestión de políticas europeas han comenzado sólo hace muy poco a ser una seria preocupación política. Mas, incluso en los debates de la $\mathrm{CIG}$, donde se esperaba que la reforma en el plano institucional estuviera en un lugar privilegiado de la agenda, apenas se pasó de manera superficial sobre el tema. Las referencias recientes, en la :Agenda 2000, a la necesidad de la reforma de la Comisión son una indicación bienvenida del interés oficial (Comisión Europea, 1997). Sin embargo, están aún muy restringidas en su alcance por la miope visión de la gestión como un subordinado de las políticas que no requiere, por tanto, más que una acción ejecutiva rutinaria.

A pesar de estas significativas limitaciones, sería erróneo hacer de la Comisión el chivo expiatorio de lo que son deficiencias del sistema profundamente arraigadas. La Comisión es tan sólo una parte constituyente de un sistema único de gobierno internacional que ha realizado avances sin precedentes en la ampliación de objetivos de la integración. Pero la fuerza del sistema es también su debilidad. Una importante fuente del déficit de gestión es el prejuicio estructural dentro del sistema total de la Unión Europea a asumir más responsabilidades de las que puede llevar a cabo con eficacia. El proceso político europeo está más vinculado a la iniciativa política que a la eficacia de las políticas. Existe una tendencia estructural a subestimar las capacidades necesarias para hacer que el sistema trabaje eficazmente. Delors, especialmente en el primer tramo de su presidencia, desarrolló una gran habilidad en potenciar la fortaleza del sistema pero prestó poca atención al tratamiento de la debilidad de su infraestructura de gestión. Durante los años noventa se ha hecho cada vez más evidente que se requiere un nuevo estilo de liderazgo que relacione la estructura con la estrategia de forma más sistemática de como se ha hecho en el pasado (CHANDLER, 1962; MAJONE, 1997). De lo contrario, en vez de respuestas constructivas a los nuevos desafíos, este sistema ahiperactivon continuará prometiendo más de lo que puede dar en temas tan importantes como el desempleo y se vinculará más con una política retórica, lo que acabará por minar su legitimidad y su eficacia.

Fue para luchar contra esta situación que Jacques Santer enfatizara la necesidad de actuar menos para actuar mejor. Las reformas están aún en proceso de gestación y dado que la Comisión es una "recién llegada" a la reforma de la gestión pública existe el peligro de que se sienta tentada por lo que parece ser la solución a corto plazo de imitar los cambios que los gobiernos nacionales han adoptado. El recurso a la imitación posee el atractivo político de parecer que ofrece una rápida solución. Los conceptos son familiares, las técnicas están disponibles y los consultores están incluso demasiado preparados para implementarlas. Pero es importante resistirse a las presiones de copiar las prescripciones de .la Nueva Gestión Pública. o Reinventing Government (OSBORvE y GAEBIER, 1992). Incluso en el nivel nacional, las reformas pretendidas de adelgazamiento. de los sistemas establecidos están resultando ser poco adecuadas para hacer frente a las necesidades de gobierno (METCALF, 1994; Peters y Savoie, 1996). Pero en el nivel europeo, los pro- 
blemas son cualitativamente diferentes. La solución no es sólo una cuestión de ponerse al nivel de las administraciones nacionales. El déficit de gestión de la Unión Europea plantea problemas sin precedentes de innovación en la organización y en la gestión. El problema no es tanto la mejora de la eficacia operacional de un sistema existente como la construcción de nuevos sistemas de gobierno que se extiendan a todos los Estados miembros y desarrollen las capacidades de gestión necesarias para asegurar su coherencia, fiabilidad y eficacia. Más que un adelgazamiento de los sistemas establecidos, se requiere un salto cuantitativo:en el desarrollo de sistemas de gobiemo nuevos, multinacionales, y asentados en la pluralidad de niveles.

\section{Reforma estructural y resistencia al cambio}

La raíz del problema actual la encontramos en que a la UE se le ha quedado pequeño su sistema institucional pero aún no ha encontrado otro más apropiado dentro del cual se puedan desarrollar las capacidades de gestión que en la actualidad se necesitan. El sistema acrual fue diseñado en los años cincuenta para que sirviera a las limitadas necesidades de seis Estados miembros, relativamente homogéneos, recién embarcados en un proceso de integración. Los cambios institucionales desde entonces han sido marginales, a pesar del sustancial incremento del acquis communautaire y el aumento del número de miembros. Nuevas funciones y responsabilidades han ido añadiéndose sin ser seguidas de alteraciones del marco básico o de los incrementos correspondientes en la capacidad de gestión. Ulteriores expansiones sin una reforma estructural no son ya factibles. Por motivos prácticos, el sistema ha alcanzado los límites de desarrollo incremental. Resulta ya inadecuado para las necesidades cada vez mayores de quince Estados miembros ahora, más heterogéneos, que han ido mucho más allá de lo que se preveía hace una década en el camino de la integración. Adjcionalmente, como consecuencia del final de la guerra fría, este sistema dado de sí en exceso, no sólo debe enfrentarse a la profundización, sino también a la ampliación. Este es un objetivo inmediato y no un panorama alejado en el tiempo, dado que la conclusión de la CIG desencadenó una nueva dificultad en torno a las negociaciones para la ampliación con los países de la Europea central y del este. El doble reto de profundización y ampliación enfrenta a la UE con la necesidad de una reforma estructural fundamental.

Como en cualquier proceso de cambio, además de importantes aspectos sobre los objetivos de la reforma y dirección de la misma, hay que considerar las cuestiones de la fuerza y la naturaleza de la resistencia al cambio. Cualquier intento de introducir un cambio a gran escala encontrará sin duda una gran oposición. A veces la oposición es manifiesta y el conflicto abierto. En otras ocasiones, existe una resistencia pasiva más sutil, pero que consigue minar la implementación del cambio. La resistencia a las reformas no debe ser considerada necesariamente como irreflexiva o injustificada. El mantenimiento de cualquier sistema requiere algo de resistencia a generar interna y externamente una transformación. Al igual que los mecanismos de defensa que desarrolla la persona o el sistema de inmunidad del organismo humano, la resistencia al cambio es un rasgo inherente de todas las organizaciones y sistemas políticos. Cuanto mayor sea la amenaza al interés vital y a los valores nucleares, más posible es que se activen fuertes mecanismos de rechazo al cambio a fin de proteger y defender la integridad del sistema. Las reformas estructurales que implican irremediables cambios en la distribución del poder y de la responsabilidad requieren una particular y cuidadosa conducción. Un proceso de reforma bien gestionado debe conseguir un delicado equilibrio entre el mantenimiento del ímpetu de cambio y la capacidad de ofrecer respuestas a las ansiedades y temores de aquellos que resultarán afectados. Con frecuencia, el éxito en las reformas depende en última instancia del compromiso y la cooperación que logren suscitar y, en el nivel europeo, ello significa convencer y persuadir a los Estados miembros. Cabría esperar el mismo tipo de reacción en España ante, por ejemplo, la presentación de propuestas para una mayor autonomía regional y para una redefinición del papel estatal.

La resistencia al cambio puede ser, sin embargo, exagerada y crear rigideces que impidan la adopción de respuestas adaptativas y causen perjuicios a largo plazo. La resistencia institucional al cambio es no sólo una inercia pasiva frente al cambio que pueda ser superada a través de la aplicación de fuerzas que acrúen como contrapeso. Es lo que ScHON (1970) denominaba "Conservadurismo dinámico". Las organizaciones y los sistemas políticos movilizan recursos activamente para resistir ante el cambio. El dynamic conservatism puede tomar la forma de subrepticiamente neutralizando o desviando más que de abierta y frontal oposición. Se pueden hacer pequeñas concesiones a fin de evitar hacer grandes cambios. La superación del *conservadurismo dinámico. es un problema particularmente agudo en determinados momentos del proceso de cambio estructural dado que implica el olvido de prácticas asentadas y la reforma de las reglas de juego establecidas. La tendencia de los líderes políticos es normalmente la de buscar caminos para preservar y alargar la vida de la estructura a la que están acostumbrados, más que la de embarcarse en aventuras de transformación estructural de peligrosos e impredecibles resultados. Los problemas estructurales son percibidos con frecuencia erróneamente como problemas de naturaleza incremental, que difieren tan sólo en grado y no en tipo de los problemas que pueden ser resueltos 
a través de medios políticos ordinarios, como la búsqueda de compromisos que satisfagan los intereses particulares sin alterar radicalmente el statu quo. De esta forma, a menudo nos encontramos ante la situación de no encontrar los apoyos necesarios para una acción colectiva hasta que los problemas alcanzan las proporciones de una crisis.

La evolución de la UE de Maastricht I a Maastricht II ilustra las dificultades para el cambio estructural y los obstáculos que crea el conservadurismo dinámico. Los términos del debate sobre la integración europea, intergubernamentalismo versus supranacionalismo, ponen de manifiesto los elementos asociados al fenómeno de transferencia de poder desde los Estados miembros hacia las instituciones comunitarias, porque en la política de la integración, las diferentes posiciones al respecto entre los propios Estados miembros han constituido el mayor obstáculo al cambio. En 1991, Maastricht I estuvo al borde del colapso porque la presidencia holandesa intentó (e ignominiosamente fracasó) forzar a un compromiso hacia una Europa federal a los Estados miembros más contrarios y hostiles a esa idea. El eventual cambio en el corazón de las instituciones fue percibido como una amenaza directa a sus soberanías nacionales. Como consecuencia, la ratificación del Tratado de Maastricht reflejó la fragilidad del apoyo político para la integración y los verdaderos peligros de desintegración. Los preparativos de la CIG 96 reabrieron algunas de estas viejas heridas y durante sus trabajos se pusieron de manifiesto las dudas y divisiones sobre el rumbo futuro que debería darse a la integración. Pero el gobierno holandés había aprendido algunas lecciones importantes de su anterior experiencia traumática, y en el último tramo de Maastricht II, una mucho más cauta presidencia holandesa hizo un importante esfuerzo para manejar las sensibles cuestiones institucionales y, especialmente, evitar el potencialmente explosivo tema del federalismo. Políticamente, el tema no estaba maduro para asegurar la aceptación de propuestas radicales para la reforma institucional. Por lo que se refiere a la integración europea, el problema de la reforma institucional no ha sido eludido, la búsqueda de una solución ha sido postpuesta. Ningún progreso podría haberse realizado en ninguna dirección si la etiqueta del federalismo hubiera ido unida al intento de reforma.

Si bien la resistencia al cambio es a menudo presentada como obstruccionismo fundado en una irreflexiva defensa de intereses creados, también podría desempeñar un papel positivo al tener la virtud de hacer hincapié sobre temas y problemas a los que los entusiastas defensores de la reforma han dado menor prioridad o prestado insuficiente atención. Un compromiso prematuro hacia una solución concreta puede cegar a los reformadores sobre los defectos o las debilidades de la solución propuesta. En el campo de la integración europea, la fijación de un determinado modelo de reforma se convierte en obstáculo del cambio. De alguna manera, Maastricht marcó el punto en que, no sólo el ritmo de la integración o la forma precisa de llevarla a cabo, sino la propia trayectoria de la integración llegaron a estar cuestionadas. Siguiendo a Deursch, et al. (1968), resulta útil distinguir entre las dos trayectorias básicas de la integración. Una trayectoria de amalgama y una trayectoria pluralista que se pueden asociar a los dos claros modelos de gobiemo europeo. El modelo ortodoxo de integración se imbricaría en la trayectoria de amalgama. Tradicionalmente, eprogresos en integración se ha medido de dos formas diferentes. En primer lugar, de la transición de formas de cooperación intergubernamental débiles y limitadas a modelos más articulados de procesos de decisión conjunta y de políticas supranacionales. En segundo término, por la gradual construcción de un Estado federal europeo, semejante a un proceso de construcción de una nación en que los gobiemos y las administraciones de los Estados miembros irían gradualmente fundiéndose (Bulmer, 1993). Ello implicaría una transferencia de poderes y de responsabilidades ejecutivas al centro de las instituciones comunitarias. Hacer que este sistema funcione eficazmente, requeriria considerables incrementos en recursos humanos y financieros en el centro y la creación de una gran función pública federal. En el momento actual, las capacidades centrales son muy limitadas, por lo que el sistema debería descansar sobre un sistema de mando convencional y un sistema de control jerárquico de gobierno para eliminar el déficit de gestión. La concepción de ‘trayectoria pluralista• de la integración se dirige en una dirección completamente diferente que no implica la fusión en un Estado unitario. En vez de ello, esta visión supone que los Estados miembros conservan sus identidades separadas y su autonomía en algunas áreas de la política al tiempo que comparten responsabilidad para la gestión común de otras áreas. Consecuentemente, su eficacia dependería de un mayor compromiso en el gobiemo y la gestión europea por parte de los Estados miembros y de sus funcionarios. La trayectoria pluralista requiere más implicación nacional, no menos. Tanto en el desarrollo de políticas como en su implementación, serían necesarias nuevas formas de redes de gestión de políticas para asegurar que el sistema funcionara eficazmente. En vez de imitar modelos nacionales de administración federal, una trayectoria pluralista requerirá innovación en los métodos de gobierno a fin de reducir el déficit de gestión.

El principal argumento de este trabajo es que la etapa entre Maastricht I y Maastricht II marca el traslado de la trayectoria de amalgama a la trayectoria pluralista. Contemplado desde la óptica convencional, ello puede ser visto como una débil forma de intergubernamentalismo, inferior a un sistema federal, pero también puede contemplarse como la emergencia de un nuevo y genuino tipo de organización política en la cual se necesita de esfuerzos creativos para diseñar y desarrollar formas de gobierno fiables y efectivas que respeten el carácter pluralista del sistema. Un factor clave para la clasificación de la UE dentro 
de la trayectoria pluralista es que no tiene competencia legal en el campo de la administración pública como tal. Posee competencias en determinadas políticas pero, en el ejercicio de las mismas, sus poderes dependen y continuarán dependiendo de la cooperación con las administraciones de los Estados miembros. Con muy pocas excepciones, la Comisión Europea debe trabajar con y a través de las administraciones nacionales (incluyendo las infraestatales) en el desempeño de su papel de gestión. La administración pública europea es un ejercicio práctico de administración comparada. Los líderes y asesores políticos en el nivel europeo se enfrentan al reto de desarrollar políticas que tienen que ser implementadas en un conjunto de países con sistemas administrativos muy diferentes. De igual modo afrontan el desafio de la capacidad de desarrollo institucional. La introducción de políticas europeas no es simplemente una cuestión de "reprogramación" de las maquinarias administrativas nacionales con las instrucciones de las nuevas políticas. Las capacidades son desiguales en los distintos países y los campos donde se desarrollan las políticas son, en ocasiones, inadecuados. Al objeto de implementar las políticas, las organizaciones existentes pueden requerir adaptaciones o la creación de nuevas organizaciones en aquellos puntos del sistema donde se detecte un vacío. El problema característico de la gestión pública europea es el de asegurar que exista una capacidad de gestión interorganizativa que abarque a todos los Estados miembros, lo cual reabre el tema del federalismo. Lo que resulta estar apareciendo en Europa es una nueva forma de federación entre naciones. El debate ideológico sobre "eurofederalismo" se ha centrado siempre, de forma casi exclusiva, en el apoyo o la oposición a la forma de Estado federal, pero un Estado federal es sólo una de las posibles fórmulas de gobierno federal (BURGESS y GaGNON, 1993). Desde una perspectiva comparativa, no hay duda de que la UE está evolucionando como un sistema federal, pero como una nueva forma de federalismo no estatal. Esto nos enfrenta al criterio general de federalismo, una combinación de autogobierno y gobiemo compartido en un sistema de múltiples niveles de gobierno (ElazAR, 1987, 1995), sin tener por qué llegar a alcanzar los atributos de la categoría de Estado. Como Willian Wallace ha observado, ala mayoría de los que han escrito sobre el proceso de toma de decisiones europeo en términos de múltiples niveles de gobierno, ha eludido la utilización del lenguaje del término "federalismon. Dado que, no obstante, la distribución de funciones entre los diferentes niveles de gobierno dentro del marco de las instituciones y regulaciones es la esencia del gobierno federal, la diferencia entre las analogías federales explícitas e implícitas, carece de importancia al menos para los académicos. (WALLACE, 1996, 459).

\section{Del federalismo a la flexibilidad}

Una cauta y circunspecta presidencia holandesa rehuyó la amenaza de la crisis en Amsterdam evitando cualquier referencia al federalismo y concentrándose más bien en la búsqueda de formas de incremento de la flexibilidad. En todo caso, el apoyo para la creación de un Estado federal europeo, unos Estados Unidos de Europa, se ha desvanecido y la oposición a esta idea ha ido creciendo ya desde la anterior presidencia. Existe un escepticismo creciente sobre la idoneidad de la transferencia de soberanía nacional a "Bruselas". Sin prestar atención sobre si ello es visto como un sueño o como una pesadilla, la visión de una Europa federal parece ahora más remota y menos realista como objetivo político. Ello, por supuesto, no elimina el problema de las reformas estructurales en el gobierno europeo si bien cambia los términos del debate. Con el federalismo claramente fuera de la agenda debido a su capacidad para crear divisiones entre Estados, la necesidad de rechazar un estancamiento propició que se prestara atención a la flexibilidad. La forma en que evoluciona el apoyo a la noción de flexibilidad y cómo se le ha ido dotando gradualmente de significado operativo en el curso de la CIG nos permite formarnos una certera idea sobre la politica de la integración y las limitaciones de la CIG. Flexibilidad ha significado un alejamiento del principio establecido en la integración europea, según el cual todos los Estados miembros participan enteramente en todos los aspectos de la integración. Aunque este principio no ha sido nunca mantenido completamente en la práctica, lo cierto es que la uniformidad constituía el estándar de referencia. Las desviaciones y las excepciones que se produjeran en el sistema eran suficientemente explicadas y justificadas. La introducción de la flexibilidad como principio de gobierno europeo supone, por tanto, un reconocimiento y a la vez una legitimación de la diversidad de los diseños de la integración europea. Resulta importante meditar sobre la integración flexible desde dos puntos de vista: por la necesidad política del momento de atraer interés sobre el concepto y por las implicaciones institucionales y administrativas que pudieran derivarse para el gobierno europeo.

En primer lugar, resulta obvio que las motivaciones políticas fueron primordiales a la hora de convertir la idea de eflexibilidad. en un buen principio de intercambio. Flexibilidad es una idea compleja y esquiva y hay que admitir que ello forma parte de su atractivo político. Se definió y fue entendida de formas diferentes y fue asumida por razones en ocasiones contradictorias. En políica, no es inusual la consecución de acuerdo en las acciones sin estar de acuerdo en los objetivos. Distintas políticas pueden concordar en medios para lograr diferentes fines. La 
amplitud de aceptación que el concepto de flexibilidad recabó durante los debates de la $\mathrm{CIG}$, se debió, al menos en parte, al hecho de que se pensaba que serviría para motivaciones políticas distintas e incluso divergentes. La flexibilidad fue percibida como un medio para resistir al cambio en la dirección de una mayor integración y, al mismo tiempo, como instrumento para superar la resistencia a tal cambio. Mientras el entusiasmo francés o alemán estaba fundado en el deseo de lanzar el proceso de integración hacia una nueva, la intención británica era la de proteger el derecho a optar por permanecer fuera de «enredos* no deseados. La flexibilidad suministraba una manera de evitar el conflicto sobre el federalismo salvando los obstáculos institucionales de la integración y descongelando actitudes politicas atrincheradas. Construyendo sobre el trabajo preparatorio de italianos e irlandeses, la presidencia holandesa vio factible la posibilidad de conseguir un nuevo consenso sobre la idea de integración flexible.

Aunque el consenso era novedoso, las ideas no lo eran tanto. Algunas de las opciones de integración flexible habian sido discutidas durante años. Para entender la forma en que el debate sobre flexibilidad se desarrolló es necesario explicar previamente algunas claves sobre los términos y las distinciones utilizadas. La flexibilidad está estrechamente asociada a lo que se ha venido en llamar integración diferenciadas. La integración diferenciada, en contraste con la práctica estándar de la integración sin diferenciación, permite la plasmación de la diversidad y la heterogeneidad de los Estados miembros de la Unión Europea. A pesar de los desacuerdos que puedan existir sobre su concepto y su práctica, esto al menos parece ser de general aceptación por todos como punto de partida. La mayor parte de las propuestas para incrementar la flexibilidad implican la adopción de algún modelo de integración diferenciada. Ello no significa, sin embargo, que la integración diferenciada sea una fórmula o una solución lista para abordar el problema de suministrar mayor flexibilidad. Más bien, es un término omniabarcante (umbrella term) que acoge una gran variedad de formas específicas y estrategias de integración. No resulta fácil determinar el número de opciones que existen en la actualidad debido a que en muchos de los términos utilizados para describirlas acaban de ver la luz. Algunos se refieren a supuestos específcos de integración diferenciada mientras que otros simplemente añaden confusión terminológica. Al efecto de establecer una base sistemática para el análisis de políticas y de elección politica, ha sido necesario clasificar y codificar los distintos tipos de integración diferenciada.

SтUвB (1996) ha intentado arrojar luz sobre esta :indigestión semántica. resultado de la proliferación de nuevos conceptos y nuevas etiquetas, identificando tres principales dimensiones de integración diferenciada: integración a varias velocidades, geometria variable e integración a la carta. La integración a varias velocidades permite la flexibilidad cuando la integración se realiza a través de un camino común. Países diferentes pueden marchar a diferente velocidad por un mismo camino. La integración a geometria variable permite la flexibilidad para quien integra un campo concreto de políicas. El mismo grupo de Estados no necesita implicarse en exactas acciones en las diferentes áreas. La integración a geometría variable avanza en diferentes direcciones con diferentes combinaciones de Estados miembros participantes. La integración a la carta tiene que ver con los diferentes campos de las políticas en que los Estados particularmente deciden involucrarse. Aquí, la atención se centra en las diferentes elecciones que los Estados miembros realizan sobre cada materia a fin de integrarse en ella o permanecer fuera. El común denominador de estos tres tipos de integración diferenciada es que las tres crean opciones de diferentes tipos que los Estados miembros pueden ejercer. Todas consideran la flexibilidad equivalente al incremento de libertad de elección de los Estados miembros para participar o no en una mayor integración: estancarse, quedar rezagado o al margen. Las tres dimensiones son analíticamente distintas en el sentido de diferenciarse tanto por el momento en que se produce la integración, como por la determinación de qué Estados miembros participan y cuáles lo hacen en el momento presente. Pero no son completamente independientes las unas de las otras. Una integración más «a la carta. debilitará los esfuerzos para promover la geometría variable o la integración a varias velocidades. Se han realizado sugerencias variadas sobre la posible combinación de esos modelos con el sistema existente basado en un núcleo unitario de políticas y competencias, a fin de permitir la diferenciación en una o más direcciones de acuerdo a las preferencias de los Estados miembros (cf. CEPR, 1995).

En segundo lugar, mientras la flexibilidad produce los beneficios políticos de evitar conflictos y diluir tensiones, las disparidades y contradicciones que produce tienen costes administrativos. Los variados tipos de flexibilidad recién descritos requieren configuraciones institucionales y capacidades de gestión diferentes para poder hacerlos funcionar. Si los gobiemos individuales no son libres para «optar por quedarse o salir en una iniciativa concreta de integración, podría producirse una vuelta a la "euroesclerosis". Pero, el permitir una u otra forma de flexibilidad crea un equilibrio inestable dado que incrementa la complejidad de la gestión política europea. Una Europa *a la carta. podría crear un confuso solapamiento de políticas comunitarias y regímenes dispares que carecería de la capacidad de actuación eficaz. Un modelo más complejo de integración diferenciada que combinara elementos de dos o más de los tipos presentados anteriormente debería corresponderse con una fórmula más compleja de gobierno europeo. Más que reducir el déficit de gestión, el efecto inmediato de un fuerte movimiento de intensificación de flexibilidad sería el de incrementarlo. 


\section{Flexibilidad en el Tratado de Amsterdam: ¿satisfaciendo las demandas o incrementando la oferta?}

El Tratado de Amsterdam contiene cláusulas específicas sobre el tema de la flexibilidad. Dichas disposiciones intentan ampliar el alcance de la "cooperación reforzada. entre los Estados miembros en el futuro. Es la primera vez se incluye una base legal para la integración flexible. Las disposiciones se incluyen en el Apéndice de esta presentación. La flexibilidad permitida es de tres tipos. La primera y más importante es la contenida en cláusulas habilitantes que permiten a los Estados miembros que estén en condiciones y deseen hacerlo, avanzar en campos específicos de las políticas, las cuales, no obstante, se mantienen bajo el marco institucional de la UE. El segundo tipo es la flexibilidad caso-por-caso que permite la abstención constructiva. Esto significa que un gobierno puede abstenerse y excluirse de la implementacón de una decisión si bien acepta que el acuerdo alcanzado por los otros compromete a la Unión. La tercera es la flexibilidad predefinida que está escrita en el Tratado y dispone la existencia de situaciones diferentes entre los Estados miembros tan pronto como el Tratado entre en vigor.

Estas disposiciones tienen dos rasgos importantes. De un lado, están muy limitadas en cuanto a su alcance. No abren un amplio campo de posibilidades para la integración difenciada sino que, más bien, son de ámbito reducido y parten de la premisa de que la flexibilidad es un último recurso. Un segundo rasgo es que la flexibilidad, en términos prácticos, equivale a ofrecer opciones, unas elecciones limitadas y condicionales para los Estados que les permiten optar. por estar dentro o fuera de campos específicos de la integración. Aunque ello pueda ser visto como una ruptura con la tradición, es una ruptura muy modesta. Tanto los temores como las esperanzas sobre el impacto del incremento de la flexibilidad son exageradas. La hipótesis de base es que la flexibilidad debe ser una excepción más que una regla. La cooperación reforzada tiende a hacer más eficiente el proceso de integración existente más que a transformarlo. El mensaje básico es simple y atractivo: libertad y elección para los Estados miembros. Los gobiernos que quieran llevar más lejos el proceso de integración deberán ser libres para hacerlo. No deberán ser retenidos por el resto de países que no quieran participar en el avance. A la inversa, dentro de los mismos límites, a los gobiernos que no quieran participar en nuevos proyectos deberá permitírseles ejercer esa opción. No deberán ser presionados o compelidos a participar. Por otro lado, un cam- bio de opinión en un momento posterior no deberá impedir su participación. Quizás la mejor forma de entender estas disposiciones es viendo cómo han sido desarrolladas desde la perspectiva del «Consejo de Ministros. Ellas reflejan la preocupación de los Estados miembros pero revelan, asimismo, la duda sobre la determinación sobre el grado de flexibilidad de que se dispone y el impacto que su ejercicio podría tener en la frágil estructura del sistema como un todo. No deben leerse como una atrevida y nueva estrategia en la integración. Más bien deben interpretarse como la nítida versión del seguro de un automóvil, con limitaciones y exclusiones mayores que la libertad de elección. Volviendo sobre lo dicho con anterioridad sobre la resistencia al cambio, estas disposiciones ilustran la operación del proceso de conservadurismo dinámico que únicamente lleva a cabo ajustes marginales de las prácticas establecidas.

El debate académico sobre integración diferenciada y el debate político en la CIG sobre la cooperación reforzada se centran ambos en cómo enfrentarse a la oferta de flexibilidad. La flexibilidad ha sido asimilada a la libertad de elección de los Estados miembros. Si existe o no una capacidad para satisfacer esas demandas es una cuestión que no se ha planteado. Si las demandas de los Estados miembros reclamando más flexibilidad se incrementan, ¿existen medios para afrontarlas?, ¿de dónde viene la oferta de flexibilidad?, iquién provee de ella y cómo se regula su asignación? Resulta confuso determinar si la limitada respuesta dada a las demandas presentadas de una mayor flexibilidad no es deseable porque la provisión de flexibilidad no sirve los propósitos de largo plazo de la integración o más bien es imposible debido a que el suministro de flexibilidad está demasiado limitado para permitir más que cambios marginales respecto de la práctica estandarizada. Es en consideración de la oferta de flexibilidad y lo que su aumento puede implicar, que surgen conexiones importantes entre los problemas de cambio estructural, eficacia y déficit de gestión. Sin embargo, previamente a abordar estos temas generales de la trayectoria de la integración, merece la pena destacar un número de desarrollos en la UE de fecha anterior a Amsterdam que no fueron discutidos en la Conferencia y tienen la potencialidad de incrementar la oferta de flexibilidad si son desarrollados sistemáticamente. Hasta ahora, han sido vistos como más o menos adiciones ad boc al modelo ortodoxo, opciones extras que respondieron a una particular necesidad, remediaron alguna carencia, o superaron alguna debilidad específica. Pero desde una perspectiva más amplia, pueden ser percibidas como pioneros de un sistema pluralista de integración. Muchos de estos elementos nos resultan ya familiares. Sin entrar demasiado en el detalle, se mencionarán siete de ellos.

- el nuevo enfoque a la armonización técnica basado en el mutuo reconocimiento de los estándares nacionales más que en la definición unificada de los mismos; 
- la creación de la estructura en tres pilares del Tratado de Maastrich;

- la formación de un nuevo tipo de agencia reguladora europea;

- el principio de subsidiariedad;

- la aceptación del derecho de los Estados miembros de permanecer al margen en determinados aspectos de la integración (opting out);

- la Unión Monetaria como proyecto distinto y condicional de integración;

- Schengen y sus mecanismos de internalización de actividades externas.

El incremento en la oferta de flexibilidad depende del entendimiento y el desarrollo de éstas y otras formas de innovación institucional y organizativa.

Considerados individualmente, cada uno de estos avances puede ser visto meramente como un pequeño despegue del modelo ortodoxo, una excepción que confirma la regla. Tomadas en su conjunto, apuntan a un cambio significativo en la trayectoria de la integración y a la necesidad de un nuevo modelo de gobierno europeo. Dada la amplia tendencia a considerar a la UE en general y a la Comisión en particular en exceso burocratizadas, pudiera resultar sorprendente sugerir que sean ellas la fuente de innovaciones institucionales. Si bien nunca ha habido un gran plan o una política general de reforma institucional, lo que ha ido sucediendo a lo largo del tiempo es que las numerosas iniciativas independientes llevadas a cabo han creado una sinergia hacia un cambio más profundo y consistente. El modelo ortodoxo de gobiemo europeo no se ha visto desplazado pero, a lo largo de los últimos años, los elementos de un nuevo y más flexible modelo han aflorado de modo más o menos empírico. Estos elementos podrían llegar a adquirir incluso más importancia a la hora de conformar un nuevo modelo de federalismo flexible que la propia $\mathrm{CIG}$, pero, en todo caso, la escala y la complejidad de la UE en el momento actual y, ciertamente, tras la ampliación dificulta una gran transformación ad boc del sistema completo en una trayectoria pluralista. Las siguientes secciones esbozan un marco general teórico para el análisis del proceso de desarrollo de capacidades de forma más sistemática.

\section{Capacidades y complejidad}

El esquema expuesto en esta sección se obtiene del estudio del detalle institucional de la UE con la intención de suministrar una forma de analizar los factores básicos que afectan al funcio- namiento de un proceso continuo de integración. Presenta un marco general para el análisis de las materias de gobiemo, gestión y eficacia, construido sobre la distinción entre capacidades y complejidad. -Capacidades son las habilidades en la gestión, los recursos y relaciones que tienen a su disposición los actores de un proceso de integración. Complejidad. es la dificultad de las tareas que surgen en un ambiente particular y con la cual tienen que enfrentarse los actores de un proceso de integración. Para comenzar, las capacidades para la integración y la complejidad de las tareas de la misma, serán tratadas como si fueran variables continuas. Ello es, por supuesto, una simplificación extremada pero permite identificar algunos elementos fundamentales como punto de partida. A continuación, se introducirán distinciones cualitativas derivadas de la teoría de la organización, lo que sugerirá que existen unos umbrales en el crecimiento de la complejidad que están pidiendo cambios estructurales en las capacidades para asegurar la efectiva gestión de la integración.

El postulado básico de este análisis es que, para conseguir una actuación eficaz, se requiere una concordancia entre complejidad y capacidades. En otras palabras, a mayor complejidad de las tareas que crea la integración, mayor capacidad de gestión es requerida para asegurar la eficacia. En una situación empírica, nada puede garantizar que las capacidades y la complejidad estén siempre en equilibrio. Al igual que en un estudio sobre la estructura y la estrategia de la gestión empresarial, pudiera observarse un desajuste entre las capacidades que se desarrollan y las necesidades de un nuevo nivel de complejidad. De hecho, pudieran darse significativas disparidades crónicas entre ellos, como la que implica la hipótesis de un déficit de gestión. Tales disparidades tienen importantes implicaciones, tanto estáticas como dinámicas, para el funcionamiento. El diagrama que sigue permite examinar las consecuencias en términos de congruencia y los principales tipos de disparidades entre las capacidades y la complejidad.

El diagrama presenta un conjunto de posibles relaciones entre las capacidades y la complejidad. Es más sencillo comenzar con los casos más simples. La línea de $45^{\circ}$ (E) representa una serie de puntos de equilibrio donde las capacidades y la complejidad están en equilibrio. A lo largo de esta línea, las capacidades son suficientes para asegurar un eficaz funcionamiento. Adicionalmente, resulta de utilidad distinguir cuatro zonas (dos a cada lado de la línea divisoria) que tienen diferentes implicaciones para el comportamiento y la eficacia. Las zonas inmediatamente próximas, a derecha e izquierda, de $\mathrm{E}$, indican moderados desajustes entre las capacidades y la complejidad, son denominadas como :zona de confort (confort) y zona de desafío (challenge).

Aquéllas más distantes, donde los desajustes entre las capacidades y la complejidad son mayores, se denominan rona descargada. (underload) y zona de sobrecarga. (overload). Los términos son en sí mismos más o menos definitorios. 


\section{CAPACIDADES}

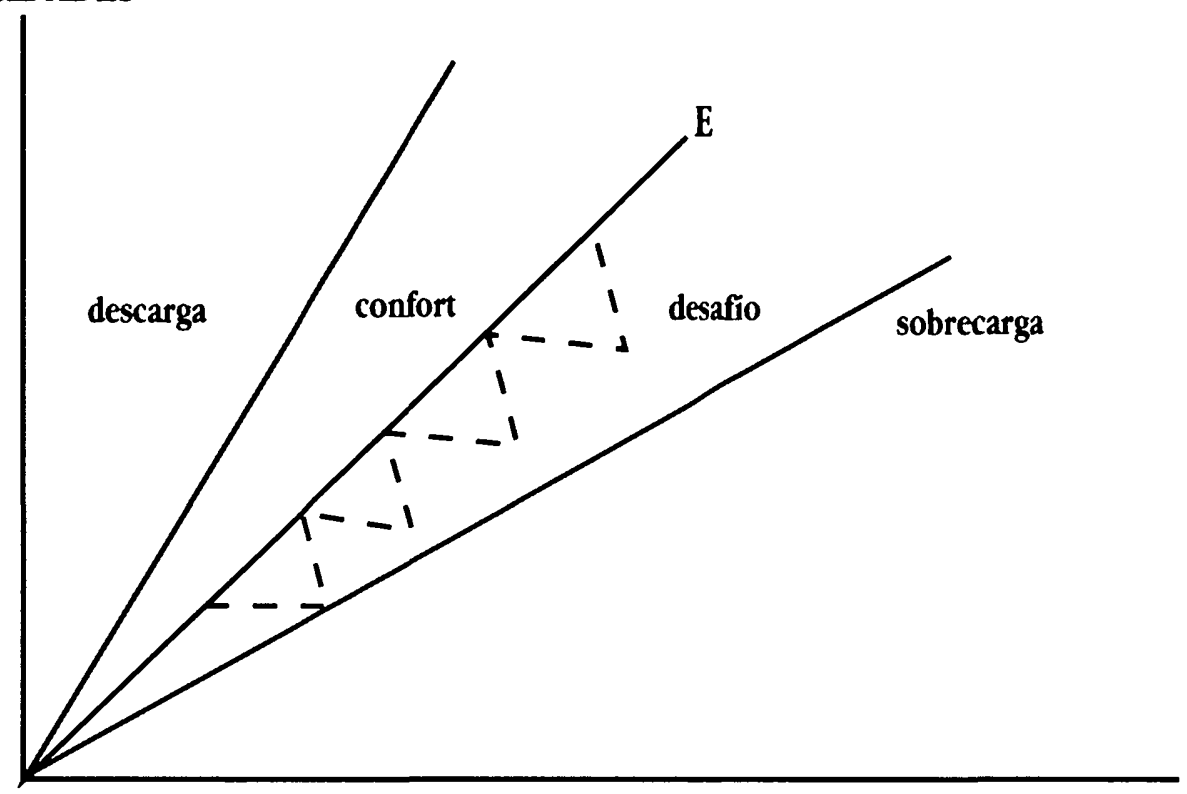

COMPLEJIDAD

En la zona de confort, las capacidades sobrepasan a las estrictamente necesarias para los estándares normales de eficacia. En este ambiente favorable, la eficacia puede ser más o menos obviada. Existen suficientes recursos organizativos para enfrentarse confortablemente a las tareas que tienen que ser llevadas a cabo. La "zona de desafio" es diferente. La mayor complejidad de las tareas de integración en relación con las capacidades disponibles conlleva la realización de un mayor esfuerzo al objeto de conseguir un funcionamiento efectivo. Las demandas son mayores de las que pueden gestionarse rutinariamente con esfuerzos normales aplicados a un repertorio establecido de procedimientos estándar. La situación implica un mayor desafío debido a la presión de las circunstancias o porque los objetivos y los niveles a los que se aspira son mayores que en los puntos de $\mathrm{E}$. Los desafíos crean un desequilibrio entre las capacidades y la complejidad que, a corto plazo, obliga a esforzarse más para mantenerse.

Observado desde una perspectiva más dinámica que estáti$\mathrm{ca}$, se constata que existen importantes diferencias entre las respuestas de comportamiento en el área de confort y en la de desafío. En el área de confort, los óptimos niveles de ejecución pueden mantenerse sin demasiada dificultad. No existe una particular motivación o incentivo para mejorar. A largo plazo, el desafío proporciona un estímulo mayor para la innovación; promueve a la realización de esfuerzos para hacer las cosas mejor en lugar de solamente continuar trabajando con aplicación. Enfrentarse a un desafío incita el progreso y refuerza las capacidades. A lo largo de un período afrontando una mayor complejidad, se observa cómo la situación conduce al de- senvolvimiento de mayores capacidades, al igual que en una actividad deportiva los resultados de un régimen de entrenamiento progresivo son las mejoras graduales en la fuerza, la habilidad o el estado físico y, por tanto, en el rendimiento. Dentro de la zona de desafío existe un estímulo a desarrollarse e innovar. Fuera de las zonas de confort y desafío los grandes desequilibrios entre las capacidades y la complejidad se traducen en una ejecución sobrecargada o descargada que afecta de forma negativa al funcionamiento.

Los factores que afectan al funcionamiento son de muy variada naturaleza según la situación sea de sobrecarga o de descarga. En la zona de sobrecarga, las capacidades son bastante inferiores de aquéllas requeridas para enfrentarse a la complejidad de las tareas. Una crisis consistiría en un deterioro en el funcionamiento y las perspectivas de vuelta a una ejecución satisfactoria disminuirían. Las crisis provocan una fuerte tensión que conducen a una situación de bloqueo, a un pobre uso incluso de las capacidades que, aunque inadecuadas, están disponibles. Crisis serias ocurrirán con probabilidad cuando las capacidades de reserva están agotadas. Una situación de descarga también conduce a un deterioro en la ejecución aunque por razones completamente diferentes. El ambiente en el que se desenvuelve el desempeño de las funciones suscita una variedad y estímulo mínimos en vez de lo contrario. En los sistemas humanos, las capacidades se mantienen y se renuevan por el uso. Bajos niveles de actividades complejas provocan poco estímulo para ejercitar y mantener las capacidades disponibles. En situaciones tales como la de una industria subvencionada, una 
burocracia protegida o la del personal de algunos hospitales, el comportamiento se conviente en institucionalizado y estereotipado más que adaptable. Una complejidad menor, inicia un círculo vicioso en el que las capacidades infrautilizadas de un comportamiento atrofiado y regresivo conducen a una disminución en la eficacia.

El esquema puede utilizarse para comparar las implicaciones de las teorías neofuncionalistas, de la integración con la propuesta aquí avanzada de que la UE afronta un déficit de gestión. Las teorías neofuncionalistas describen la integración como un proceso que se autorrefuerza gradualmente y caracterizado por una sucesión de desafíos de cambio y de ciclos de desafíos de cambio. El proceso de integración sigue un camino en la "zona de desafíon, como la representada en el diagrama. Una nueva agenda de integración con objetivos ambiciosos pero realistas conduce a mayores niveles de integración. El círculo virtuoso del proceso consiste en que el enfrentamiento ante retos suministra las capacidades adicionales para lanzar la siguiente fase. Establecido en estos generales términos, el neofuncionalismo es compatible con la interpretación de una evolución incremental del proceso de integración. Existen unos niveles garantizados de integración. Pero, tal y como muestra la historia, no existe garantía de que la integración se vaya a mantener automáticamente dentro de la zona de desafío. En distintos momentos, el proceso se ha estancado o incluso ha retrocedido cuando la evolución se ha bloqueado. En otras ocasiones, se han producido crisis cuando un incremento demasiado rápido de demandas en el sistema ha sobrecargado sus capacidades limitadas e inflexibles. De hecho, debido a la dificultad en la reorganización de los recursos, es bastante factible en algunos campos de políticas europeas una experiencia de sobrecarga, desafio, confort y descarga simultáneamente.

La hipótesis optimista de la teoría neofuncionalista es que el ímpetu de la integración estimulará el desarrollo de las capacidades adicionales necesarias para asegurar la eficacia. Por el contrario, defender la teoría del edéficit de gestión. implica estar convencido de que las capacidades no se desarrollan espontáneamente al ritmo de los nuevos compromisos. El proceso de instauración de nuevos objetivos políticos y el de desarrollo de capacidades de gestión necesarias para llevarlos a cabo no están tan estrechamente unidos como para asegurar un funcionamiento eficaz. Por un lado, existen tendencias institucionales hacia las nuevas iniciativas que incrementan la complejidad. Asimismo, existen importantes presiones en el proceso de clesarrollo de nuevas capacidades. Como resultado, el sistema es muy propenso a tender a la crisis y la sobrecarga.

\section{Integración en los campos de "turbulencia"}

El peligro de la sobrecarga es más serio de lo que la discusión precedente pudiera mostrar, dado que la reducción del déficit de gestión no sólo se resolvería a través de un incremento cuantitativo de las capacidades de gestión. En tiempos de cambio estructural, la demanda de capacidades de gestión es más sofisticada. Niveles elevados de complejidad implican el desarrollo de formas de gobierno cualitativamente diferentes para poder afrontar la amenaza de la turbulencia. El término turbulencia es a menudo utilizado de forma vaga para describir una situación de dificultad. En teoría de la organización, tiene un significado preciso que se relaciona con una particular complejidad en las condiciones ambientales. Resulta útil comenzar distinguiendo entre ambientes organizativos de diferentes niveles de complejidad. Las diferencias están definidas, para redes pluralistas de organizaciones, como distinciones en la textura causal del ambiente organizativo (EMERY y TRIST, 1965; METCAIFE, 1974; HAAS, 1976). La textura causal del ambiente es propiedad de un sistema pluralista en su conjunto. Está influenciado por y a su vez influye en el comportamiento de las organizaciones constituyentes. Las diferencias en la extensión de la interdependencia alteran las condiciones de eficacia organizativa e influyen en la ejecución del sistema completo de organizaciones. Más en detalle, cuando la interdependencia organizativa es baja, la eficacia organizativa está altamente determinada por la eficacia intraorganizativa dentro de los condicionamientos impuestos por el ambiente exterior de modo que la ejecución del sistema interorganizativo en su conjunto es el agregado del nivel de funcionamiento de las partes. Cuando la interdependencia aumenta, la eficacia de las organizaciones individuales comienza a depender cada vez más de su coordinación con otras organizaciones y se establecen redes interorganizativas fiables.

Se pueden distinguir cuatro tipos de ambientes organizativos con texturas causales de complejidad creciente: 1) estable homogéneo; 2) estable diferenciado; 3) con perturbaciones reactivas; 4) turbulento. La transición de unas condiciones de perturbación reactiva a la turbulencia resulta tener relevancia particular para el caso de la UE en su actual etapa de desarrollo. El ambiente de turbulencia, que es el de mayor dificultad desde el punto de vista de la gestión, ha sido, generalmente, el principal foco de atención en el debate teórico. 


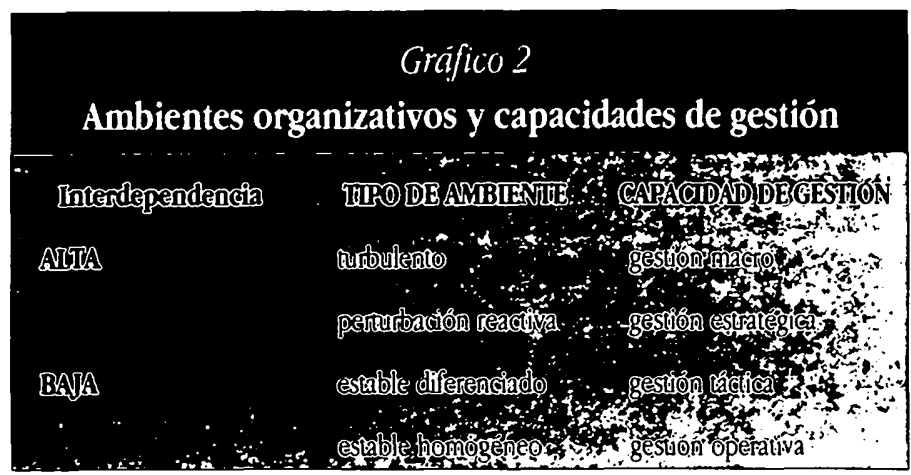

Estas distinciones permiten una afinación y una especificación teórica del marco de capacidades/complejidad esbozado más arriba. Los tipos de ambiente organizativo identifican una serie de umbrales o discontinuidades que deben abordarse con el desarrollo de nuevos y distintos tipos de capacidad organizativa, si los niveles de acruación se quieren mantener y mejorar. En el nivel más bajo, sólo se necesitan capacidades de gestión operativa para actuar con eficacia en un ambiente estable y no diferenciado. En el tipo 2 la eficacia en el ambiente requiere unas capacidades de gestión táctica a fin de descubrir oportunidades y explotar las colocaciones de los ecological niches. En ambientes de "perturbación reactiva" (tipo 3), las capacidades de gestión estratégica se hacen necesarias para conseguir eficacia. En este tipo de ambiente, las acciones organizativas desencadenan reacciones externas y contrarreacciones que deben ser anticipadas y tenidas en consideración en el proceso de formulación estratégica. Tanto desde el punto de vista teórico como desde el práctico, es el tipo 4 ambiente de turbulencia el que centra la mayor atención. Además de la atracción speriodística que pudiera provocar el término, la turbulencia indica un tipo peculiar de dificultad del ambiente de la organización, caracterizado por altos niveles de interdependencia organizativa. En tal tipo de entornos, la eficacia de las organizaciones individuales depende en extremo de lo bien que coordinen sus actividades con otras organizaciones. Un fracaso en la gestión constructiva de la interdependencia puede tener consecuencias devastadoras no sólo para la actuación de las organizaciones individuales sino también para el sistema en su conjunto.

Los rasgos distintivos de un ambiente de turbulencia tienen una relevancia directa en la discusión sobre la flexibilidad en el proceso de integración europea y pudiera ser también especialmente relevante para un país como España que está planificando la realización de reformas estructurales en su forma de gobernar. Los ambientes del tipo 4 se describen como turbulentos" porque las organizaciones individuales, al actuar independientemente, no sólo no están capacitadas para asegurar su propia eficacia, sino que al intentar proteger sus propios intereses ante cambios impredecibles son las responsables del empeoramiento de la situación. Sus propias acciones amplifican y refuerzan las tendencias a la desintegración. La turbulencia implica cambios sistemáticos que son literalmente inmanejables por las organizaciones individuales en el nivel micro. Ello nos da una pista sobre qué tipo de capacidades se requieren para gestionar una situación de turbulencia: las capacidades de gestión macro requeridas para transformar el marco de gobiemo. Es importante destacar al respecto que los procesos inmersos en la gestión de una situación de turbulencia son el reverso de aquellos otros eficaces en ambientes de menor complejidad. El proceso dominante es macro-micro en vez de micro-macro. Depende enormemente del desarrollo de capacidades de gestión interorganizativa para la reorganización al nivel del sistema en su conjunto. Considerando que la gestión estratégica se centra en la intensificación de la ventaja competitiva de las organizaciones individuales en el nivel micro, el problema en los ambientes de turbulencia potencial es el de reforzar las capacidades para colaborar en la gestión de las reorganizaciones a gran escala y los cambios estructurales en el nivel macro. Por poner un importante y tópico ejemplo, en la políica de pesca existe claramente un déficit de gestión en el que no existen estas capacidades. El interés competitivo a corto plazo de las flotas pesqueras está reñido con el interés colectivo a largo plazo de la industria en su conjunto. La sobrecapacidad conduciría irremediablemente al agotamiento de las reservas pesqueras. Pero el régimen pesquero europeo carece de las capacidades necesarias en el nivel macro para gestionar un cambio estructural con eficacia. La industria podrá ocasionar su propio derrumbamiento al igual que sus acciones provocarán el colapso de las reservas pesqueras.

Por lo general, el tránsito del ambiente del tipo 3 uperturbaciones reactivas" al tipo 4 "turbulencia" conlleva unas demandas muy especiales en cuanto a las capacidades de gobierno y de gestión. Existe una discontinuidad extremadamente significativa (y no una progresión lineal) entre las capacidades necesarias para asegurar la eficacia en un ambiente de perturbaciones reactivas y en un ambiente de turbulencia. En cierto modo, los procesos implicados en la gestión de la turbulencia son el reverso de aquellos que se emplean para gestionar los contextos menos complejos. El cambio cualitativo en la naturaleza de los problemas demanda una respuesta armonizada a través del desarrollo de capacidades para una reorganización sistemática. Los modelos desarrollados por los teóricos de la catástrofe nos ofrecen una herramienta muy útil para visualizar las distintas implicaciones. Al inicio de la turbulencia, los requerimientos básicos para enfrentarse a ella pueden ser representados en un modelo de catástrofe cúspide. Los factores de control son (i) las capacidades para la acción colectiva con la configuración especial de un sistema de macrogestión y (ii) los niveles de complejidad alrededor de los umbrales donde existen más probabilidades de producción de problemas. Tal y como muestra el diagrama, la eficacia de la respuesta a los problemas estructurales depende 
enormemente de si las capacidades de macrogestión son desarrolladas para afrontar el desafío del cambio estructural. Si esas capacidades ya existen, podrán activarse (a-b). De no existir, deberá llevarse a cabo una estrategia deliberada para conformar dichas capacidades al objeto de asegurar su desarrollo con carácter previo a que surjan los problemas estructurales. Si no se realizan esfuerzos para desarrollar tales capacidades (e-f) o las respuestas son inadecuadas (e-c-d), los fallos a la hora de mitigar los efectos de la discontinuidad de los problemas que deben ser resueltos (de incrementales a estructurales) y de un cambio cualitativo en las capacidades de gestión (de micro a ma- cro), se plamarán en una desintegración periudicial y no en una integración exitosa. Al margen de la relevancia que pudiera representar para el federalismo en Europa, este análisis podría igualmente aplicarse a las relaciones entre los diferentes niveles de gobiemo en España. El éxito de las reformas en los sistemas de gobiemo de múltiples niveles depende en gran medida de la producción de innovaciones institucionales que refuercen las relaciones de colaboración entre las organizaciones, de forma que cuando surjan problemas estructurales, puedan trabajar conjuntamente como cuerpos coordinados de un sistema de macroges tión.

\section{Gráfico 3}

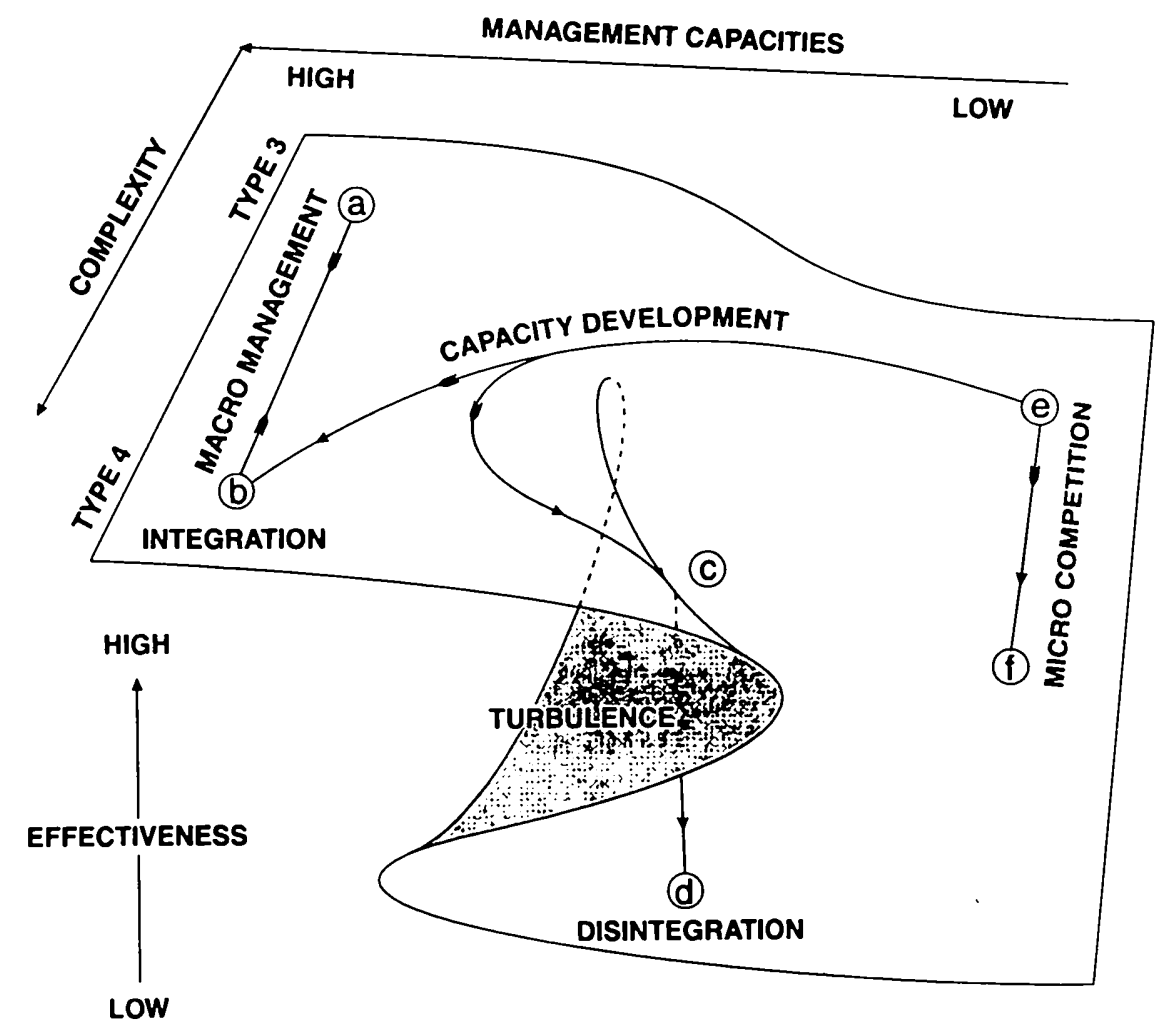

\section{Conclusiones}

La urgencia de abordar el déficit de gestión de la UE se fundamenta en la amenaza de desintegración que afronta un sistema ya de por sí altamente complejo y de gran turbulencia potencial. La corrección de la propensión estructural de la Unión
Europea a asumir más tareas de las que puede realizar, exige un refuerzo de construcción de capacidades. La incipiente turbulencia exige una respuesta concertada y un programa bien planificado de acción coordinada a fin de poder gestionar el cambio estructural a un nivel macro. La consecución de la eficacia dependerá fundamentalmente de si las capacidades de gestión macro son adecuadas para la tarea de dirigir el cambio estructural. Si no lo son la Unión Europea se desintegrará en la línea de los 
análisis familiares del dilema del prisionero y el pánico competitivo o de los problemas de acción colectiva en los cuales, lo que es individualmente racional, es colectivamente irracional.

Gestionar, en condiciones de sturbulencian exige grandes inversiones en cooperación interorganizacional en el nivel macro a fin de transformar el ambiente en el que la actividad del nivel micro se desarrolla. Ello no significa centralización. Tampoco significa que las instituciones a nivel europeo deban asumir las funciones de las administraciones nacionales. Una trayectoria pluralista sobre integración implica el establecimiento de múltiples niveles en los procesos de políticas y en las instituciones a través de los cuales se puedan superar los problemas estructurales. Está de moda en estos días en el campo de la gestión empresarial hablar de learning onganization. En el campo de la integración europea es cada vez más importante desarrollar esas capacidades de aprendizaje al nivel interorganizativo a fin de hacer frente a las necesidades del federalismo flexible.

\section{A. Cláusulas generales que se incluirán como nuevo titulo en las disposiciones comunes del TUE}

\section{Artículo 1}

1. Los Estados miembros que tengan la intención de instiruir una cooperación más estrecha entre sí podrán hacer uso de las instituciones, procedimientos y mecanismos establecidos en los Tratados siempre que esa cooperación:

a) pretenda fomentar los objetivos de la Unión, así como proteger y servir sus intereses;

b) respete los principios contenidos en los Tratados y el marco institucional único de la Unión;

c) se use sólo como último recurso cuando no se hayan podido alcanzar los objetivos de los Tratados por medio de los procedimientos pertinentes establecidos en los mismos;

d) implique al menos la mayoría de los Estados miembros;

e) no afecte al acervo comunitario ni a las medidas adoptadas a tenor de las demás disposiciones del Tratado;

f) no afecte a las competencias, derechos, obligaciones e intereses de los Estados miembros que no participen en ella;

g) esté abierto a todos los Estados miembros y permita a dichos Estados participar en la cooperación en cualquier momento, siempre que acaten la decisión de base y las decisiones tomadas en ese contexto; h) cumpla los criterios adicionales específicos previstos en el artículo $5 \mathrm{~A}$ del TCE y en el artículo K.12 del presente Tratado, según el ámbito de que se trate, y esté autorizada por el Consejo con arreglo a los procedimientos establecidos en ellos.

2. Los Estados miembros aplicarán, en la medida en que les corresponda, los actos y decisiones adoptados para la ejecución de la cooperación en la que participen. Los Estados miembros que no participen en dicha cooperación no impedirán su aplicación por parte de los Estados miembros que participen en ella.

\section{Articulo 2}

1. A efectos de la adopción de los actos y decisiones necesarios para la ejecución de la cooperación a que se refiere el artículo 1, serán de aplicación las disposiciones institucionales pertinentes en los Tratados. No obstante, si bien todos los miembros del Consejo podrán participar en las deliberaciones, sólo aquellos que representen a los Estados miembros participantes en dicha cooperación tomarán parte en la adopción de decisiones; la mayoría cualificada se definirá como la misma proporción de los votos de los miembros del Consejo interesados en la cooperación, ponderados de acuerdo con el apartado 2 del artículo 248 del Tratado constitutivo de la Comunidad Europea; la unanimidad quedará constituida únicamente por los miembros del Consejo interesados en la cooperación.

2. Los gastos resultantes de la aplicación de la cooperación diferentes de los gastos administrativos ocasionados por las instiuciones correrán por cuenta de los Estados miembros participantes, salvo que el Consejo decida otra cosa por unanimidad.

\section{Articulo 3}

El Consejo y la Comisión informarán periódicamente al Parlamento Europeo del desarrollo de la cooperación más estrecha establecida sobre la base del presente título.

\section{B. Cláusulas especificas del TCE}

\section{Artículo 5 A TCE}

1. Con arreglo a lo dispuesto en los artículos (1) y (2) ${ }^{\prime}$, se podrá autorizar a los Estados miembros que se propongan instituir entre sí una cooperación más estrecha a hacer uso de las instituciones, procedimientos y mecanismos establecidos por el presente Tratado, siempre que la cooperación que se proponga:

a) no se refiera a ámbitos que sean de exclusiva competencia de la Comunidad; 
b) no afecte a las políticas, acciones o programas comunitarios;

c) no se refiera a la ciudadanía de la Unión ni establezca una discriminación entre nacionales de los Estados miembros;

d) permanezca dentro de los límites de las competencias atribuidas a la Comunidad por el presente Tratado; y

e) no constituya una discriminación ni una restricción del comercio entre los Estados miembros y no falsee las condiciones de competencia entre ellos.
2. El Consejo concederá la autorización contemplada en el apartado 1 pronunciándose por mayoría cualificada, a propuesta de la Comisión y previa consulta al Parlamento Europeo.

Si un miembro del Consejo declara que, debido a importantes y expresas razones de políica nacional, se propone oponerse a la concesión de una autorización por mayoría cualificada, no se llevará a cabo la votación. El Consejo, por mayoría cualificada, podrá pedir que el asunto se remita al Consejo Europeo para decidir sobre él por unanimidad.

\section{Notas}

Ariculo traducido por Carmen Navarro GOMEZ.

' Cláusulas generales sobre una cooperación más estrecha.

- Profesor de Gesión Pública y Director de investigación del Instituro Europeo de Administración Pública. Maastricht (Holanda).

\section{Bibliografía}

Simon J. Burmer, -The Governance of the European Union: A New Institutionalist Approach, Journal of Public Policy, 13(4), October-December 1993: págs. 351-380.

Michel BuRGESS and Alain-G. GAGNON, Comparative Federalism and Federation-Competing traditions and future directions. Herfordshire: Harvester Vheatsheaf, 1993.

CEPR, Flexible Integrtion: Towards a More Effective and Democratic Europe. London: Centre for Economic Policy Research, 1995.

Alfred D. Jr. Cahndlek, Strategy and Structure. Cambridge, MA.: MIT Press, 1962.

Karl W. Deutsch; Sidney A. BuRRel; Robert A. Kan:, Maurice LeE Jr.; Matin LICHTERMAN; Raymond E. LNDDGREN; Francis L. LOEWENHEM y Richard W. VAN WAGENEN, Political Community and the North Atlantic Area (Princeton, New Jersey: Princeton University Press, 1968).

EuRopenv COMmission, Agenda 2000 For a stronger and wider Union. Bulletin of the European Union, Supplement 5/97 (Document drawn up on the basis of COMO7) 2000 final), Luxembourg: Office for Official Publications of the European Communities, 1997.

Daniel J. Elazar, Exploring Federalism. Tuscaloosa: University of Alabama Press, 1987.

Daniel J. Euzar, From Statism to Federalism: A Paradigm Shift, Publius: The Joumal of Federalism, 25(2), Spring 1995: pázs. 5-18.

Fred E. EmerY y Eric L. TrIST, The Causal Texture of Organizational Enviroments, Human Relations 18, February, 1965: páys. 21-32.

Emst B. Hits, Turbulent Fields and the Theory of Regional Integration, International Organizations 30(2), Spring, 1976: págs. 173-172.
Giandomenico MAjone, -From the Positive to the Regulatory State: Causes and Consequences of Changes in the Mode of Govemance, To appear in Joumal of Public Policy, October 1997.

Les Metcalfe, Systems Models, Economic Models and the Causal Texture of Organizational Environments: An Approach to Macro-Organization Theory, Human Relations, Vol 27 (7), September, 1974: págs. 639-663.

Les MetCalfe, •After 1992: Can the Commission Manage Europe?•, Australian Joumal of Public Administrations 51(1), March 1992: págs. 117-130.

Les MetCALfE, IIntemational Policy Co-ordination and Public Management Reform., Intemational Review of Administrative Sciences, London, Thousand Oaks and New Delhi: Sage, Vol. 60, N.² 2, 1994: págs. 271-290.

Les METCalfe, la Comisión Europea como una organización-red, Gestión y Análisis de Politicas Priblicas (1995), Número 4, Septiembre/Diciembre 1995, págs. 25-36.

David OsBorve y Ted GAEBLER, Reinventing Government, Reading, MA: Addison-Wesley, 1992.

Guy B. PETERS y Donald J. SAvOIE, Managing Incoherence: The Coordination and Empowerment Conundrum, Public Administration Review, May/June 1996, Vol. 56, N.8 3: págs. 281-289.

Donald A. SCHON, Beyond the Stable State, W.W. Norton, New York, 1971.

Alexander C.G. STUBB, A Categorization of Differentiated Integration, Joumal of Common Market Studies, Vol. 34, N.², June 1996: págs. 283-295.

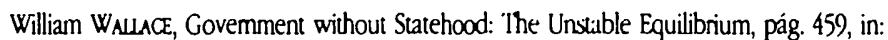
Helen Wallace and William Wallace (eds.) Policy Making in the European Union, Oxford University Press, Oxford, 1996. 
